\title{
$O$ acometimento renal na hipertensão arterial e diabetes mellitus tipo 2: como identificar e prevenir? A visão do cardiologista
}

\author{
The renal involvement in hypertension and type 2 diabetes mellitus: \\ how to identify and to prevent? A vision of the cardiologist
}

Érika M. G. Campana

Andréa A. Brandão*

\section{Resumo}

A hipertensão arterial (HA) e o diabetes mellitus tipo 2 (DM2) são fatores de risco cardiovascular que frequentemente coexistem. Estas condições clínicas têm grande impacto epidemiológico, com elevados custos médicosociais e econômicos. Muito comumente estas duas condições cursam com algum grau de lesão renal e representam as principais causas de doença renal terminal. Por tudo isto o controle da pressão arterial (PA), da glicemia e dos lípides séricos são fundamentais para prevenir ou postergar o desenvolvimento da nefropatia diabética. O benefício do controle glicêmico mais intenso na prevenção da nefropatia diabética e de complicações micro e macrovasculares não está completamente estabelecido. De forma semelhante, evidências de literatura têm demonstrado benefício da redução da PA para valores de 130-135/80 mmHg em diabéticos. No entanto, reduções mais intensas da PA apresentam evidências limitadas. $\mathrm{O}$ tratamento da HA e do DM deve envolver mudanças de hábitos de vida e tratamento medicamentoso concomitantemente. Todas as classes de anti-hipertensivos são capazes de reduzir a PA e, portanto contribuir para a prevenção da nefropatia diabética; entretanto, destacam-se os benefícios dos bloqueadores do sistema renina angiotensina aldosterona. A escolha do esquema hipoglicemiante será baseada nos valores da glicemia de jejum, pós-prandial e da HbAlc, no peso e idade do paciente, na presença de complicações associadas e na interação com outras drogas. Os autores discutem neste artigo as metas de tratamento da PA e da glicemia, as linhas gerais de tratamento não medicamentoso e medicamentoso da HA e do DM, bem como as principais estratégias para proteção renal, na visão do cardiologista.

Descritores: Hipertensão; Diabetes mellitus; Nefropatias diabéticas. 


\section{Abstract}

Hypertension and type 2 diabetes are cardiovascular risk factors that frequently coexist. These clinical conditions have great epidemiologic impact, high-cost medical-social and economic. Very often these two conditions occur with some degree of renal injury and is the leading cause of nephropathy terminal. For all this the control of blood pressure, blood glucose and serum lipids are essential to prevent or at least delay the development of diabetic nephropathy. The benefit of more intensive glucose control in preventing diabetic nephropathy and macrovascular and microvascular complications is not fully established. Similarly, evidences from literature have demonstrated the benefit of reducing BP values for 130-135/80 mmHg in diabetics. However, larger decreases in $\mathrm{BP}$ have limited evidence. The treatment of hypertension and DM should involve lifestyle changes and drug therapy concomitantly. All classes of antihypertensive drugs are able to reduce the PA and therefore contribute to the prevention of diabetic nephropathy, however, highlight the benefits of blockers of the renin angiotensin aldosterone system. The choice of hypoglycemic scheme will be based on the values of fasting glucose, postprandial and HbAlc, weight and age of the patient, the presence of associated complications and the interaction with other drugs. The authors discuss in this article the goals of treatment for BP and blood glucose, the outlines of drug and non-drug treatment of hypertension and DM, as well as key strategies for renal protection, in view of the cardiologist.

Keywords: Hypertension; Diabetes mellitus; Diabetic nephropathies.

\section{Epidemiologia da associação de hipertensão arterial e diabetes tipo 2}

O número de indivíduos hipertensos e diabéticos tem aumentado em todo o mundo. ${ }^{1-6} \mathrm{~A}$ prevalência global de hipertensão arterial (HA) está estimada entre 20 a 30\%. ${ }^{1}$ A prevalência do diabetes mellitus (DM) na população brasileira é da ordem de 7,6\%;,7 mundialmente projeta-se que no ano de 2030 cerca de 300 milhões de indivíduos serão diabéticos. ${ }^{4-7}$

Essas duas patologias frequentemente se associam e são acompanhadas de complicações graves, que impactam em aumento de custos econômicos e sociais para o indivíduo e para o sistema de saúde. ${ }^{1-6}$ Dados americanos demonstraram um aumento de quase 3 vezes nos custos em saúde de pacientes portadores de nefropatia diabética (ND) quando comparados aos diabéticos sem nefropatia. ${ }^{8}$ No Brasil, dados do DATASUS de 2007, reportam um gasto anual estimado de cerca de 2 bilhões de reais para a terapia de substituição renal. ${ }^{9}$

Cerca de um terço dos pacientes com DM desenvolvem ND; ela é a principal causa de doença renal terminal e está associada ao aumento da mortalidade geral e cardiovascular. ${ }^{4-6}$ Os controles glicêmico, pressórico e lipídico inadequados, a presença de hábitos de vida indesejáveis, como o tabagismo e a alta ingestão de proteínas na dieta, e a presença de valores mais elevados de albuminúria (mesmo dentro dos parâmetros de normalidade), associada à predisposição genética, são elementos fundamentais na fisiopatologia dessa doença. Existe uma relação íntima e direta entre a elevação da pressão arterial e o surgimento da ND. ${ }^{1-6}$ Cerca de $40 \%$ dos pacientes já são hipertensos na ocasião de seu diagnóstico de DM tipo $2 .^{4-6}$ Os estudos têm mostrado que o controle da pressão arterial (PA) é primordial para a prevenção da ND; resultados do estudo UKPDS ${ }^{10,11}$ demonstraram que, para cada $10 \mathrm{mmHg}$ de diminuição na pressão sistólica média, houve uma diminuição significativa de $13 \%$ do risco de complicações microvasculares.

Por tudo isso, o controle da PA, da glicemia e dos lípides séricos são fundamentais para prevenir ou, no mínimo, postergar o desenvolvimento da ND. ${ }^{1-6}$

\section{Metas de pressão arterial e glicemia}

Alcançar as metas de controle glicêmico e pressórico é fundamental para prevenir a instalação da nefropatia diabética. ${ }^{1-6} \mathrm{O}$ objetivo 
primordial no tratamento do DM deve ser o alcance de valores glicêmicos tão próximos da normoglicemia quanto possível, ou seja, os níveis de hemoglobina glicada (HbAlc) devem ser os menores possíveis, sem aumentar o risco de hipoglicemia. ${ }^{4-6}$ Já o objetivo do tratamento da HA é reduzir a PA e consequentemente as complicações associadas a essa condição clínica. ${ }^{1-3}$

$\mathrm{O}$ dilema que envolve a determinação da meta ideal do controle metabólico a ser alcançada na prevenção de complicações macro e microvasculares nos diabéticos ainda não foi solucionado..$^{4-6} \mathrm{O}$ benefício de um controle glicêmico mais intenso na prevenção da ND não está completamente estabelecido na literatura. ${ }^{4-6}$ Em um grupo de participantes do UKPDS ${ }^{11}$ selecionado para permanecer em seguimento por 10 anos após a conclusão do estudo, o controle mais intenso e precoce da glicemia (HbAlc: 7,0\% vs.7,9\%) foi acompanhado de redução de complicações microvasculares, incluindo a insuficiência renal (redução de risco de 24\% em 10 anos; $\mathrm{p}=0,001$ ). No estudo ADVANCE ${ }^{12}$ o grupo com controle glicêmico mais intenso (HbA1c: 6,5\% vs. 7,3\%) apresentou uma discreta diminuição dos novos casos de microalbuminúria $(23,7 \%$ vs. $25,7 \% ; \mathrm{p}=0,02)$, do surgimento de macroalbuminúria ( $2,9 \%$ vs. $4,1 \%$; $\mathrm{p}<0,001)$ e de ND $(4,1 \%$ vs. $5,2 \%$; $\mathrm{p}=0,006)$. Resultados semelhantes foram observados no estudo VADT $^{13}$ com redução da progressão de micro para macroalbuminúria $(2,9 \%$ vs. $5,1 \% ; \mathrm{p}=0,04)$ quando se alcançava um controle glicêmico mais intenso (HbA1c: 6,9\% vs. 8,4\%). Entretanto, não

Tabela 1. Metas de controle glicêmico recomendadas pela Sociedade Brasileira de Diabetes ${ }^{4}$

\begin{tabular}{ccc}
\hline Parâmetro & Metas* & Nível de evidência \\
\hline HbA1c & $<7 \%$ & $B$ \\
\hline Glicemia de jejum & $<110 \mathrm{mg} / \mathrm{dl}$ & $\mathrm{B}$ \\
\hline Glicemia pré-prandial & $<110 \mathrm{mg} / \mathrm{d}$ & $\mathrm{B}$ \\
\hline Glicemia pós-prandial & $<140 \mathrm{mg} / \mathrm{dl}$ & $\mathrm{B}$ \\
\hline
\end{tabular}

$\mathrm{HbA1c}=$ hemoglobina glicada.

$B=$ estudos experimentais e observacionais de menor consistência.

*As metas devem ser alcançadas sem aumentar o risco de hipoglicemia. foram observadas diferenças significativas para os desfechos renais maiores como duplicação da creatinina basal e redução da taxa de filtração glomerular (TFGE) abaixo de $15 \mathrm{ml} / \mathrm{min}$ neste último estudo. Finalmente, o encerramento precoce do estudo ACCORD ${ }^{14,15}$ suscitou importante discussão acerca do controle glicêmico muito intenso. Em uma análise post hoc o controle glicêmico mais intenso (HbA1c: $<6 \%$ vs. $<7-7,9 \%)$ reduziu a incidência de ND $(4,1 \%$ vs. $5,2 \%$; RR: 0,$79 ; \mathrm{p}=0,006$ ), porém houve um aumento dos episódios de hipoglicemia severa com o tratamento mais agressivo (2,7\% vs. $1,5 \%$; RR: 1,$86 ; p<0,001)$ e, ainda, essa estratégia foi preditora de mortalidade (RR: 1,2; IC 95\%: 1,01 a 1,$46 ; p=0,04)$ e não reduziu as complicações cardiovasculares.

Fica claro que alcançar a meta glicêmica precocemente e manter o controle em longo prazo reduzindo a hiperglicemia crônica é uma estratégia fundamental para prevenção da microalbuminuria e sua progressão para a ND. Entretanto, este controle não deve envolver metas excessivamente agressivas sob pena de aumentar os riscos de hipoglicemia e complicações cardiovasculares. ${ }^{4-6}$ Dessa forma, a Sociedade Brasileira de Diabetes ${ }^{4}$ recomenda como metas glicêmicas a serem alcançadas em pacientes adultos diabéticos as listadas na tabela 1 .

Conjuntamente ao controle metabólico, o tratamento da HA nos pacientes diabéticos tem importância crucial para redução das complicações macro e microvasculares. ${ }^{1-6}$ Sabidamente, a elevação progressiva da PA a partir de 115-75 mmHg está associada ao aumento da morbidade e mortalidade na população geral, incluindo os diabéticos, e valores de pressão arterial sistólica (PAS) superiores a $120 \mathrm{mmHg}$ estão associados à ocorrência de doença renal terminal nessa população. ${ }^{1,4,5}$ Evidências de literatura têm demonstrado benefício (redução de eventos coronarianos, acidente vascular cerebral e nefropatia) da redução da pressão sanguínea para valores de 130-135/80 mmHg nesses pacientes. ${ }^{16}$ Por outro lado, a mostra de 


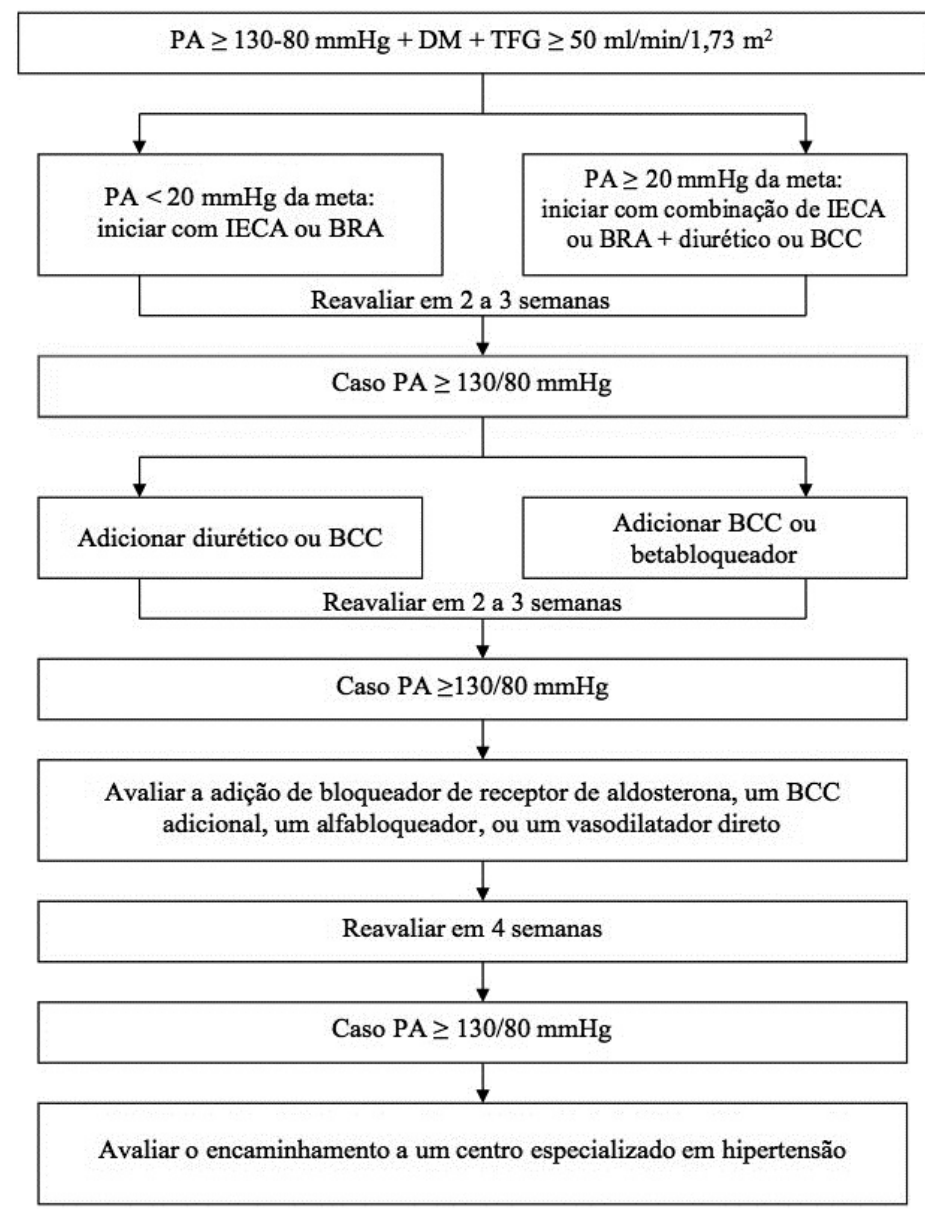

\section{Figura 1. Algoritmo de abordagem de pacientes hipertensos diabéticos ${ }^{8}$}

benefícios para reduções mais intensas da PA é limitada. ${ }^{1,4,5,7}$

As primeiras evidências de que o controle da PA teria um papel-chave na prevenção das complicações do DM foram demonstradas pelo clássico estudo UKPDS, ${ }^{17}$ no qual o grupo com menor PA (144-82 mmHg vs. $154-87 \mathrm{mmHg}$ ) apresentou menor ocorrência de eventos cardiovasculares $(\mathrm{p}<0,0001)$, porém sem qualquer benefício na prevenção da ND. Salienta-se que os valores adotados neste estudo são muito superiores aos atualmente recomendados.

Subsequentemente 4 importantes estudos randomizados discutiram a questão: o HOT Study, ${ }^{18}$ uma nova edição do UKPDS, ${ }^{10}$ o estudo $\mathrm{ABCD}^{19}$ e o estudo $\mathrm{ACCORD}^{15}$ e sugeriram que uma redução mais intensa da PA era capaz de prevenir complicações macro e microvasculares nos pacientes diabéticos.
No HOT Study ${ }^{18}$ (PAD < 80 vs. 90 mmHg) os resultados demonstraram redução de eventos cardiovasculares nos diabéticos com o controle mais agressivo da PA (redução 51\%, p = 0,005), entretanto não houve diferenças significativas para os valores de creatinina entre os dois grupos ao final de 3,8 anos de estudo.

$\mathrm{O} \mathrm{UKPDS}^{10}$ foi desenvolvido para determinar o impacto de uma estratégia mais agressiva de controle da PA na prevenção do desenvolvimento de doença renal terminal em diabéticos tipo 2. Ao final de 8,4 anos observou-se uma redução de $37 \%$ nos eventos microvasculares no grupo com controle mais agressivo da PA (PA $<150-80 \mathrm{mmHg}$ ). Vale reforçar que este valor de PA é muito superior ao recomendado atualmente.

$\mathrm{O} \mathrm{ABCD}^{19}$ também testou o impacto de uma estratégia mais agressiva de controle da PA na prevenção de eventos macro e micro- 
vasculares em DM2. Não houve diferença estatisticamente significativa na complicação renal (clearance de creatinina) entre os grupos (média de PA 128-75 mmHg vs. 137-81 mmHg).

Já no estudo ACCORD, ${ }^{15}$ os diabéticos foram randomizados para 2 grupos de tratamento (PAS $<120 \mathrm{mmHg}$ vs. PAS $<140 \mathrm{mmHg}$ ). Os resultados mostraram que não havia benefício na redução do desfecho primário (morbimortalidade cardiovascular) $(\mathrm{p}=0,20)$ com a redução mais intensa da PA e, ainda, o grupo de tratamento mais intensivo apresentava um aumento da ocorrência de efeitos colaterais $(p=0,001)$. $\mathrm{O}$ desfecho secundário microalbuminúria foi significativamente menor no grupo tratamento intensivo $(p=0,009)$ sem, no entanto, haver diferença na taxa de filtração glomerular entre os 2 grupos de tratamento ao final de 5 anos.

Ensaios clínicos mais recentes nesse campo, apontam para a possível existência de uma curva em "J” para a PA e a ocorrência de eventos cardiovasculares. Os principais estudos sobre metas de PA no DM concluíram que a totalidade da evidência disponível atualmente não justifica a redução da PA menor que 130-80 $\mathrm{mmHg}$ como estratégia para retardar a progressão da doença renal, exceto em pacientes com proteinúria maior que $500 \mathrm{mg} /$ dia ou naqueles com risco muito elevado de acidente vascular encefálico onde valores mais baixos poderiam ser considerados. ${ }^{21}$ Resultados semelhantes foram observados na metanálise publicada por Bangalore e colaboradores, ${ }^{20}$ que demonstraram que as metas de PAS entre 130-135 mmHg eram as desejáveis. Quando a redução da PAS era mais intensa, menor que $130 \mathrm{mmHg}$, os autores observavam uma redução expressiva da ocorrência de acidente vascular encefálico (17\%), da albuminúria (17\%) e da ND (27\%), mas não observavam redução das complicações cardiovasculares e da taxa de progressão para doença renal terminal e havia, ainda, um aumento de $40 \%$ dos eventos adversos sérios. Dessa forma, as principais diretrizes ${ }^{1-6}$ definem como metas a serem alcançadas no controle da PA em pacientes hipertensos e diabéticos valores em torno de 130-80 mmHg.

\section{Estratégias atuais para o tratamento da PA e da glicemia}

A abordagem inicial da HA no paciente diabético envolve mudança de hábitos de vida, com adequação dos hábitos alimentares, controle do peso, prática de exercícios físicos regulares e abandono do tabagismo e do etilismo. Entretanto, por serem os diabéticos pacientes de alto risco para complicações cardiovasculares e renais, obrigatoriamente deverão receber tratamento medicamentoso para HA. Frequentemente esses pacientes necessitarão de terapêutica combinada com dois ou mais fármacos para alcançar as metas de PA propostas pelas principais diretrizes médicas. ${ }^{1-6}$

Virtualmente todas as classes de anti-hipertensivos são capazes de reduzir a PA e, portanto, contribuir para a prevenção da nefropatia diabética. Entretanto, as principais sociedades médicas recomendam que um bloqueador do sistema renina angiotensina aldosterona (SRAA) esteja incluído no esquema terapêutico de pacientes hipertensos diabéticos. ${ }^{1-6}$ Esse posicionamento decorre de evidências na literatura que apontam para os benefícios dos bloqueadores do SRAA no tratamento desse grupo de pacientes, que incluem a melhora da sensibilidade à insulina, a prevenção de complicações cardiovasculares e sua capacidade de prevenir a progressão da ND. ${ }^{1-6}$

A microalbuminúria persistente (30-299 $\mathrm{mg} / 24 \mathrm{~h}$ ) é o marcador mais precoce para o desenvolvimento da ND e é também um marcador de risco cardiovascular aumentado. ${ }^{4-6}$ A utilização de fármacos bloqueadores do SRAA tem demonstrado capacidade de retardar o início da microalbuminúria, reduzir a progressão da micro para a macroalbuminúria e a progressão para estágios mais avançados de ND. Os efeitos redutores da proteinúria com essas drogas em pacientes diabéticos ocorrem precocemente, é estável e independe da magnitude da redução da PA.4-6 Adicionalmente, estudos revelam que os individuos portadores de doença renal crônica são os que mais obtêm benefício renal e cardio- 
vascular com o uso das drogas bloqueadoras do SRAA. ${ }^{22}$ Por essas vantagens, recomenda-se o uso rotineiro desses fármacos em todos os pacientes diabéticos hipertensos. ${ }^{1-6}$ No final da década de 1990, os resultados do UKPDS $39^{23}$ já demonstravam que o uso do inibidor da enzima conversora de angiotensina (IECA), captopril, era eficaz na prevenção primária das complicações microvasculares do DM2. Na mesma época, os resultados do clássico estudo MICRO-HOPE ${ }^{24}$ reforçaram essa evidência, utilizando um diferente IECA, o ramipril, demonstrando capacidade de prevenir a evolução de micro para macroalbuminúria no DM2, com redução da nefropatia manifesta em $24 \%$; $p=0,027$. Nessa mesma direção, evidências de benefício na prevenção de complicações microvasculares com os bloqueadores dos receptores de angiotensina II (BRA) foram demonstradas com 2 representantes dessa classe, a losartana (redução de risco de doença renal terminal de 28\%; $\mathrm{p}=0,002)^{25}$ e a irbesartana (redução de risco de doença renal terminal de $23 \%$; $\mathrm{p}=0,07),{ }^{26}$ corroborando o benefício do uso de bloqueadores do SRAA na prevenção da ND.

O uso de combinação de fármacos é muito frequente nesse grupo de pacientes de alto risco cardiovascular, no qual sabidamente o controle da PA é mais difícil. ${ }^{1-3} \mathrm{O}$ estudo ADVANCE ${ }^{27,28}$ demonstrou que o uso de uma combinação fixa de um IECA (perindopril) com um diurético (indapamida) reduziu significativamente as complicações micro e macrovasculares, bem como doenças cardiovasculares e mortalidade total. Os efeitos absolutos da combinação de drogas se mostraram vantajosos. Para cada 1.000 pacientes com doença renal em estágio maior ou igual a 3 tratados por 5 anos, a combinação impediu 18 eventos cardiovasculares, 26 mortes cardiovasculares e 30 mortes por todas as causas, quando em comparação com os 6 eventos cardiovasculares, 6 mortes cardiovasculares e 10 mortes por 1.000 pacientes sem doença renal terminal no grupo em uso apenas de perindopril.

O duplo bloqueio do SRAA sugeria um racional atraente para otimizar o controle pressórico, a regressão da albuminúria e, consequentemente, a prevenção de complicações em pacientes de alto risco como os diabéticos. Essa estratégia foi testada no estudo ONTARGET ${ }^{29}$ sem benefícios cardioprotetores adicionais e com aumento da ocorrência de efeitos colaterais. Os efeitos do duplo bloqueio do SRAA na nefroproteção também não foram amplamente demonstrados, podendo ser considerados em casos selecionados nos quais há proteinúria maior que $500 \mathrm{mg} / 24$ horas. Portanto, à luz dos conhecimentos atuais, essa estratégia não deve ser utilizada de forma ampla.

Outras classes de medicamentos, como diuréticos, antagonistas de cálcio e betabloqueadores, podem ser utilizados como terapia combinada aos bloqueadores dos SRAAs para alcançar as metas pressóricas desejadas ou como terapia alternativa nos casos raros de intolerância a IECA ou BRA. ${ }^{1-6}$ Uma estratégia racional para a abordagem terapêutica da $\mathrm{PA}$ de pacientes hipertensos diabéticos está apresentada na figura 1 .

Semelhantemente ao que ocorre na HA, o tratamento do DM deve envolver mudanças de hábitos de vida, com foco principal na dieta alimentar acompanhada de controle do peso, prática de exercícios físicos regulares e abandono do tabagismo e do etilismo. Quando as medidas dietéticas não forem suficientes para o controle glicêmico adequado, a terapêutica medicamentosa deverá ser instituída. ${ }^{4-6} \mathrm{~A}$ escolha do esquema hipoglicemiante será baseada nos valores da glicemia de jejum, pós-prandial e da HbA1c; em características do paciente como peso e idade; na presença de complicações associadas e na interação com outras drogas. ${ }^{4-6}$

$\mathrm{Na}$ fase inicial da doença, onde predomina a hiperglicemia, a obesidade e a insulinorresistência, a droga de escolha é a metformina, pois não estimula secreção de insulina e não promove o ganho de peso. Na segunda fase do DM, onde ocorre diminuição da secreção de insulina, os secretagogos (sulfonilureias) estão indicados, preferencialmente em combinação 


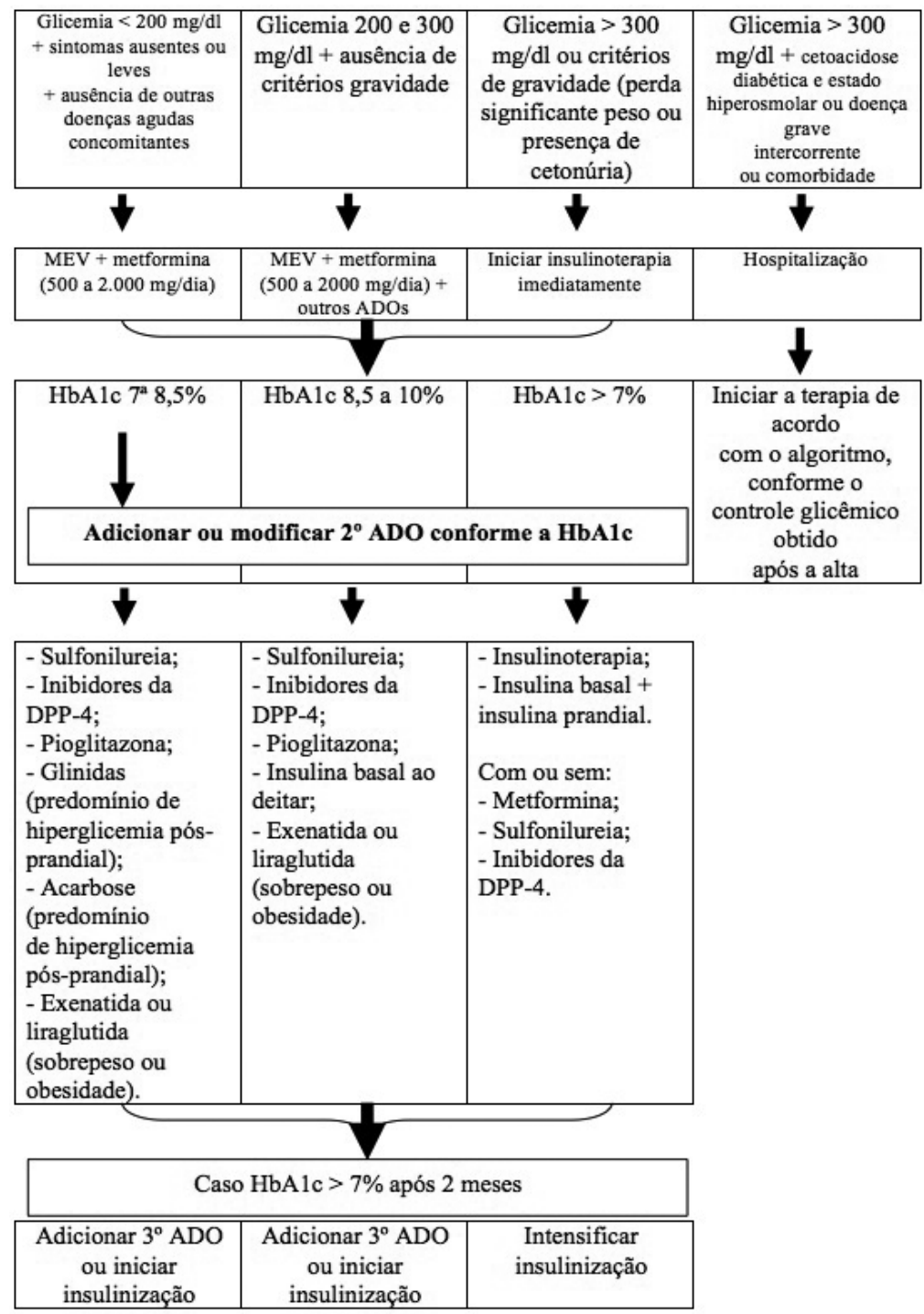

Figura 2. Algoritmo para tratamento medicamentoso do diabetes mellitus $s^{4-6}$

$\mathrm{MEV}=$ modificações de estilo de vida; $\mathrm{ADO}=$ antidiabético oral; $\mathrm{HbA1C}=$ hemoglobina glicada.

com os sensibilizadores insulínicos (glitazonas).

$\mathrm{Na}$ fase 3 (cerca de 10 anos de doença), com o agravamento da perda de secreção de insulina e com o aparecimento das complicações, está indicada o início da insulina (insulinização oportuna). Na fase 4 , o predomínio é da depleção severa de insulina e a insulinização plena está indicada (figura 2). ${ }^{4-6}$

A função renal do paciente é um parâmetro fundamental na escolha do esquema medicamentoso dos diabéticos. A metformina está contraindicada nos pacientes com TFG menor que $30 \mathrm{ml} / \mathrm{min}$, pelo risco de acidose lática. Nos pacientes com TFG entre 30 e $59 \mathrm{ml} / \mathrm{min}$ o uso da metformina deve ser cauteloso. As sulfonilureias (exceto glimepirida) são excretadas pelo rim e não devem ser utilizados em pacientes com comprometimento da função renal. A acarbose não deve ser utilizada na ND acima do estágio 3 pelo risco de lesão hepática e valores de creatinina menores que $2 \mathrm{mg} / \mathrm{dl}$ constituem contraindicação para seu uso. ${ }^{4-6}$ Como alternativas seguras para tratamento oral de portadores de ND destacam-se a repaglinida e as glitazonas. A roziglitazona tem demonstrado capacidade de reduzir a albuminúria, ${ }^{30}$ sugerindo um potencial efeito nefroprotetor. ${ }^{4-6}$

Entre os inibidores da dipeptidilpeptidase 
(DPP-4), a sitagliptina necessita ajuste de dose para portadores de ND estágios 4 e 5 . A vidagliptina não necessita de ajuste de dose para ND estágios 1 a 3 , mas está contraindicada em pacientes com doença renal estágios 4 e 5 . Finalmente, o análogo da GLP-1, exanitida, também pode ser utilizada nos estágios iniciais, mas está contraindicada nos estágios 4 e 5 da ND. ${ }^{4-6}$

\section{Referências}

1. Sociedade Brasileira de Cardiologia/Sociedade Brasileira de Hipertensão/Sociedade Brasileira de Nefrologia. VI Diretrizes Brasileiras de Hipertensão. Arq Bras Cardiol. 2010;95(1 supl.1):1-51.

2. ESC Committee for Practice Guidelines (CPG)/ ESHScientific Council. Reappraisal of European guidelines on hypertension management: a European Society of Hypertension Task Force document. J Hypertens. 2009,27:2121-58.

3. The Seventh report of joint National Committee on prevention, detection, evaluation, and treatment of high blood pressure. The JNC 7 Report. JAMA. 2003;289:2560-72.

4. Gomes MB, Lerario AC, Milech A, Angelucci AP, Golbert A, Carrilho AJF, et al. Sociedade Brasileira de Diabetes. Diretrizes da Sociedade Brasileira de Diabetes 2009. [acessado 2011 nov. 20] Disponível em: http://www.diabetes.org.br/ attachments/diretrizes09_final.pdf.

5. American Diabetes Association. Standards of medical care in diabetes - 2013. Diabetes Care. 2013 Jan; 36 (Suppl 1):S11-66. http://www.doi. org/10.2337/dc13-S011.

6. American Diabetes Association. Standards of medical care in diabetes - 2010. Diabetes Care 2010;33 (Suppl 1):S11-61. Erratum in: Diabetes Care. 2010;33 (3):692.

7. Gross JL, Nehme M. Detecção e tratamento das complicações crônicas do diabetes melito: Consenso da Sociedade Brasileira de Diabetes e Conselho Brasileiro de Oftalmologia. AMB Rev Assoc Med Bras 1999;45(3):279-84.

8. Bakris GL. Recognition, pathogenesis, and treatment of different stages of nephropathy in patients with type 2 diabetes mellitus. Mayo Clin Proc 2011 May;86(5):444-56.

9. Siviero P, Rodrigues RN, Machado CJ. Doença renal crônica: um agravo de proporções crescentes na população brasileira. Disponivel em: http://web.face.ufmg.br/cedeplar/site/ index.php?option $=$ com_content $\&$ view $=$ articl e\&id=1349:doenca-renal-cronica-um-agravode-proporcoes-crescentes-na-populacao- brasileira\&catid=191:textos-para-discussao.

10. Adler AI, Stratton IM, Neil HA, Yudkin JS, Matthews DR, Cull CA, et al. Association of systolic blood pressure with macrovascular and microvascular complications of type 2 diabetes (UKPDS 36): prospective observational study. BMJ. 2000 Aug 12;321(7258):412-9.

11. Holman RR, Paul SK, Bethel MA, Matthews DR, Neil HA. 10-year follow-up of intensive glucose control in type 2 diabetes. N Engl J Med. 2008; 359 (15):1577-89. http://www.doi.org/10.1056/ NEJMoa0806470

12. ADVANCE Collaborative Group. Intensive blood glucose control and vascular outcomes in patients with type 2 diabetes. $\mathrm{N}$ Engl J Med. 2008 Jun 12;358(24):2560-72.

13. Duckworth W, Abraira C, Moritz T, Reda D, Emanuele N, Reaven PD, et al. Glucose control and vascular complications in veterans with type 2 diabetes. N Engl J Med. 2009 Jan 8;360(2):129-39. http://www.doi.org/10.1056/ NEJMoa0808431

14. Action to Control Cardiovascular Risk in Diabetes Study Group, Gerstein HC, Miller ME, Byington RP, Goff DC Jr, Bigger JT, et al. Effects of intensive glucose lowering in type 2 diabetes. N Engl J Med. 2008;358(24):2545- 9. http://www.doi.org/10.1056/NEJMoa0802743

15. Riddle MC. Effects of intensive glucose lowering in the management of patients with type 2 diabetes mellitus in the Action to Control Cardiovascular Risk in Diabetes (ACCORD) trial. Circulation. 2010 Aug 24;122(8):844-6.

16. Lipman ML, Schiffrin EL. What is the ideal blood pressure goal for patients with diabetes mellitus and nephropathy? Curr Cardiol Rep. 2012 Dec;14(6):651-9.

17. UK Prospective Diabetes Study Group (UKPDS 39). Efficacy of atenolol and captopril in reducing risk of macrovascular and microvascular complications in type 2 diabetes. BMJ. 1998;317:713-20.

18. Hansson L, Zanchetti A, Carruthers SG, Dahlöf B, Elmfeldt D, Julius S, et al. Effects of intensive blood-pressure lowering and low-dose aspirin in patients with hypertension: principal results of the Hypertension Optimal Treatment (HOT) randomized trial. Lancet. 998;351(9118):1755-62.

19. Schrier RW, Estacio RO, Mehler PS, Hiatt WR. Appropriate blood pressure control in hypertensive and normotensive type 2 diabetes mellitus: a summary of the ABCD trial. Nat Clin Pract Nephrol. 2007 Aug; 3(8): 428-38.

20. Bangalore S, Kumar S, Lobach I, Messerli FH. Blood pressure targets in subjects with type 2 diabetes mellitus/impaired fasting glucose: observations from traditional and bayesian 
random-effects meta-analyses of randomized trials. Circulation. 2011 Jun 21;123(24):2799-810.

21. Flynn C, Bakris GL. Blood pressure targets for patients with diabetes or kidney disease. Curr Hypertens Rep 2011 Dec;13(6):452-5.

22. Ninomiya T, Perkovic V, de Galan BE, Zoungas S, Pillai A, Jardine $M$ et al. Albuminuria and kidney function independently predict cardiovascular and renal outcomes in diabetes. J Am Soc Nephrol. 2009;20:1813-21.

23. Heart Outcomes Prevention Evaluation (HOPE) Study Investigators. Effects of ramipril on cardiovascular and microvascular outcomes in people with diabetes mellitus: result of the HOPE study and Micro-HOPE Substudy. Lancet. 2000;355:253-9.

24. Brenner BM, Cooper ME, Zeeuw D, Keane WF, Mitch WE, Parving H-H, et al. Effects of losartan on renal and cardiovascular outcomes in patients with type 2 diabetes and nephropathy. N Engl J Med. 2001;345:861-9.

25. Lewis EJ, Hunsicker LG, Clarke WR, Berl T, Pohl MA, Collaborative Study Group, et al. Renoprotective effect of the angiotensinreceptor antagonist irbesartan in patients with nephropathy due to type 2 diabetes. $\mathrm{N}$ Engl J Med. 2001;345(12):851-60.

26. Patel A, ADVANCE Collaborative Group, MacMahon S, Chalmers J, Neal B. Effects of a fixed combination of perindopril and indapamide on macrovascular and microvascular outcomes in patients with type 2 diabetes mellitus (the ADVANCE trial): a randomised controlled trial. Lancet. 2007;370:829-40.

27. Heerspink HJ, Ninomiya T, Perkovic V, Woodward M, Zoungas S, Cass A, et al. Effects of a fixed combination of perindopril and indapamide in patients with type 2 diabetes and chronic kidney disease. Eur Heart J. 2010 Dec;31(23):2888-96.

28. Sleight $P$, Redon J, Verdecchia P, Mancia G, Gao $\mathrm{P}$, ONTARGET investigators, et al. Prognostic value of blood pressure in patients with high vascular risk in the Ongoing Telmisartan Alone and in combination with Ramipril Global Endpoint Trial study. J Hypertens. 2009;27(7):1360-9.

29. Iglesias P, Diez JJ. Peroxisome proliferatoractivated receptor gamma agonists in renal disease. Eur J Endocrinol. 2006;154:613-21. 